\title{
COLLAPSE OF ROTATING SUPRAMASSIVE NEUTRON STARS TO BLACK HOLES: FULLY GENERAL RELATIVISTIC SIMULATIONS
}

\author{
Masaru Shibata ${ }^{1}$ \\ 1 Graduate School of Arts and Sciences, University of Tokyo, \\ Komaba, Meguro, Tokyo 153-8902, Japan \\ Draft version August 22, 2018
}

\begin{abstract}
We study the final state of the gravitational collapse of uniformly rotating supramassive neutron stars by axisymmetric simulations in full general relativity. The rotating stars provided as the initial condition are marginally stable against quasiradial gravitational collapse and its equatorial radius rotates with the Kepler velocity (i.e., the star is at the mass-shedding limit). To model the neutron stars, we adopt the polytropic equations of state for a wide range of the polytropic index as $n=2 / 3,4 / 5,1,3 / 2$ and 2 . We follow the formation and evolution of the black holes, and show that irrespective of the value of $n(2 / 3 \leq n \leq 2)$, the final state is a Kerr black hole and the disk mass is very small $\left(<10^{-3}\right.$ of the initial stellar mass).
\end{abstract}

Subject headings: black hole physics - relativity - hydrodynamics - stars: rotation

\section{INTRODUCTION}

Neutron stars are in general rotating. Rotation can support neutron stars with higher mass than the maximum static limit, producing supramassive stars, as defined and numerically computed by Cook et al. (1992, 1994a). Supramassive neutron stars may be created (i) when neutron stars accrete gas from a normal binary companion (Cook et al. 1994b), (ii) after the merger of binary neutron stars (Shibata \& Uryū 2000, 2002), and (iii) after gravitational collapse of massive stellar core.

Since viscosity drives any equilibrium star to a uniformly rotating state, stationary neutron stars are believed to be uniformly rotating. The final state after the collapse of the marginally stable and uniformly rotating supramassive neutron stars is the subject of this paper.

Rotating neutron stars with a density higher than a critical value are unstable against gravitational collapse. Such critical density is determined using the turning point theorem (Friedman et al. 1988; Cook et al. 1992). The final state of the unstable spherical stars in the adiabatic collapse is a Schwarzschild black hole. On the other hand, in the rotating case, it is not trivial: All the fluid elements may not collapse to a Kerr black hole, leaving a fraction of the mass around the black hole to form disks. The final state after the collapse of rotating stars is one of the fundamental questions in general relativistic astrophysics.

To clarify the final state of the gravitational collapse of rotating neutron stars, numerical simulations in full general relativity are the best approach. Two groups have already performed the simulations for relativistic collapse of rotating stars (Nakamura 1981; Nakamura et al. 1987; Stark \& Piran 1985; Piran \& Stark 1986). However, they have not studied the collapse of marginally stable rotating neutron stars which are plausible initial conditions for the collapse in nature. Probably this is because numerical methods for computation of initial data sets describing rapidly rotating neutron stars, as well as numerical tools, techniques and sufficient computational resources have become available only quite recently. Over the last 15 years, robust numerical techniques for constructing equilibrium models of rotating neutron stars in full general relativity have been established (Komatsu et al. 1989; Cook et al. 1992; Salgado et al. 1994; Stergioulas 1998). More recently, robust methods for the numerical evolution of the coupled equations of Einstein's and hydrodynamic equations have been also established (e.g., Shibata 1999b, 2003; Font 2000; Font et al. 2002; Siebel et al. 2002, 2003).

In a previous paper (Shibata et al. 2000), we reported the first numerical result for the gravitational collapse, which was computed by a three-dimensional numerical implementation in full general relativity. In that paper, we adopted the polytropic equation of state with $n=1$ where $n$ is the polytropic index, and gave a uniformly rotating and marginally stable neutron star at a mass-shedding limit (at which the equator of a star rotates with the Kepler velocity) as the initial condition. The total grid number in the simulations was only $153 \times 77 \times 77$ for $x-y-z$ (we assumed the equatorial plane symmetry and $\pi$ rotation symmetry) because of the restricted computational resources at that time, and as a result, the equatorial radius (polar radius) of the neutron star is covered only by 40 (23) grid points initially. We found that the collapse leads to a black hole (we determined the location of the apparent horizon), and indicated that nonaxisymmetric instabilities do not turn on during the collapse. However, we were not able to determine the final state of the gravitational collapse because of the insufficient grid resolution.

Since nonaxisymmetric instabilities are not likely to be relevant during the collapse, the simulation should be carried out under the assumption of the axial symmetry. With this restriction, we could significantly improve the grid resolution for a given computational resource. Motivated by this fact, we have constructed a numerical code for axisymmetric numerical simulation in full general relativity, which has been already completed (Shibata 2000, 2003). Because of the restriction to the axial symmetry as well as progress in computational resources, we can easily increase the grid number $3-5$ times as large as that in the previous three-dimensional simulation (Shibata et al. 2000) even in inexpensive personal computers. As 
a result, we can search for convergent numerical results changing the grid number for a wide range with inexpensive computational cost. In addition, we adopt a highresolution shock-capturing scheme for evolving the hydrodynamic equations (Shibata 2003), which enables us to assess whether shocks play an important role during the collapse to a black hole.

In this paper, we present new numerical results for gravitational collapse computed by the new axisymmetric numerical implementation. The simulations were carried out setting marginally stable equilibrium neutron stars as the initial condition. We focus on the collapse of uniformly rotating supramassive neutron stars at mass-shedding limits as before. By exploring rotating stars at mass-shedding limits, we can clarify the final state of the collapsed objects most efficiently. To investigate the effect of the stiffness of equations of state, we adopt polytropic equations of state with a wide variety of the polytropic index. The state of marginally stable stars which is characterized by the compactness, angular momentum parameter and density distribution depends strongly on the equations of state. This implies that the final state after the gravitational collapse of rotating stars could depend strongly on the equations of state, in contrast to the collapse of nonrotating stars in which the Schwarzschild black hole with no disks is the unique outcome. To classify the type of gravitational collapse and its final state, a systematic study for a wide variety of equations of state is essential.

In Sec. 2, we briefly describe our formulation, initial data, and spatial gauge conditions. In Sec. 3, we present numerical results. In Sec. 4, we provide a summary. Throughout this paper, we adopt the units $G=c=K=1$ where $G, c$ and $K$ denote the gravitational constant, speed of light and polytropic constant, respectively. We use Cartesian coordinates $x^{k}=(x, y, z)$ as the spatial coordinate with $r=\sqrt{x^{2}+y^{2}+z^{2}} ; t$ denotes coordinate time.

\section{SUMMARY OF FORMULATION AND INITIAL CONDITIONS}

We performed hydrodynamic simulations in general relativity for the axisymmetric spacetime using the same formulation as that used in a previous paper (Shibata 2003), to which the reader may refer for details and basic equations.

We assume that neutron stars are composed of the inviscid, ideal fluid. Then, the fundamental variables for the hydrodynamic equations are:

$$
\begin{aligned}
\rho & : \text { rest mass density, } \\
\varepsilon & : \text { specific internal energy, } \\
P & : \text { pressure, } \\
u^{\mu} & : \text { four velocity, } \\
v^{i} & =\frac{d x^{i}}{d t}=\frac{u^{i}}{u^{t}}
\end{aligned}
$$

where subscripts $i, j, k, \cdots$ denote $x, y$ and $z$, and $\mu$ the spacetime components. As the fundamental variables to be evolved in the numerical simulations, we in addition define a density $\rho_{*}\left(=\rho \alpha u^{t} e^{6 \phi}\right)(\phi$ is defined below) and weighted four-velocity $\hat{u}_{i}\left(=(1+\varepsilon+P / \rho) u_{i}\right)$ from which the total rest mass and angular momentum of the system can be integrated as

$$
\begin{aligned}
M_{*} & =\int d^{3} x \rho_{*}, \\
J & =\int d^{3} x \rho_{*} \hat{u}_{\varphi} .
\end{aligned}
$$

General relativistic hydrodynamic equations are solved using the so-called high-resolution shock-capturing scheme (Shibata 2003; see Font 2002 for a general review of highresolution shock-capturing schemes) with the cylindrical coordinates.

We neglect effects of viscosity and magnetic fields. The dissipation and angular momentum transport timescales by these effects are much longer than the dynamical timescale unless the magnitude of viscosity and magnetic fields is extremely large (e.g., Baumgarte et al. 2000). Thus, neglecting them is appropriate assumption.

The fundamental variables for the geometry are:

$$
\begin{aligned}
\alpha & : \text { lapse function, } \\
\beta^{k} & : \text { shift vector, } \\
\gamma_{i j} & : \text { metric in 3D spatial hypersurface, } \\
\gamma & =e^{12 \phi}=\operatorname{det}\left(\gamma_{i j}\right) \\
\tilde{\gamma}_{i j} & =e^{-4 \phi} \gamma_{i j} \\
K_{i j} & : \text { extrinsic curvature. }
\end{aligned}
$$

As in the series of our papers, we evolve $\tilde{\gamma}_{i j}, \phi, \tilde{A}_{i j} \equiv$ $e^{-4 \phi}\left(K_{i j}-\gamma_{i j} K_{k}{ }^{k}\right)$ together with the three auxiliary functions $F_{i} \equiv \delta^{j k} \partial_{j} \tilde{\gamma}_{i k}$ and the the trace of the extrinsic curvature $K_{k}{ }^{k}$ with a free evolution code (see Shibata \& Uryu 2002 for the latest version of the formulation).

The Einstein equations are solved in the Cartesian coordinates. To impose the axisymmetric boundary condition, the so-called Cartoon method is used (Alcubierre et al. 2001b): Assuming a reflection symmetry with respect to the $z=0$ plane, we perform simulations using a fixed uniform grid with the size $N \times 3 \times N$ in $x-y-z$ which covers a computational domain as $0 \leq x \leq L, 0 \leq z \leq L$ and $-\Delta \leq y \leq \Delta$. Here, $N$ and $L$ are constants and $\Delta=L / N$. For $y= \pm \Delta$, the axisymmetric boundary conditions are imposed.

The slicing conditions are basically the same as those adopted in previous papers (Shibata 1999, 2000, 2003; Shibata \& Uryū 2000, 2002); i.e., we impose an approximate maximal slice condition $\left(K_{k}^{k} \simeq 0\right)$. On the other hand, we adopt two spatial gauge conditions for the shift vector. One is an approximate minimal distortion (AMD) gauge condition $\left[\tilde{D}_{i}\left(\partial_{t} \tilde{\gamma}^{i j}\right) \simeq 0\right.$ where $\tilde{D}_{i}$ is the covariant derivative with respect to $\tilde{\gamma}_{i j}$ ] (Shibata 1999) which has been used in our previous works. In contrast with previous papers (e.g., Shibata et al. 2000), we used the AMD gauge condition without modification. The other is a dynamical gauge condition (e.g., Alcubierre et al. 2001a; Lindblom \& Scheel 2003). In the present work, we impose the dynamical gauge condition with the equation

$$
\partial_{t} \beta^{k}=\tilde{\gamma}^{k l}\left(F_{l}+\Delta t \partial_{t} F_{l}\right)
$$

where $\Delta t$ denotes a timestep in numerical computation. The second term in the right-hand side of Eq. (5) is introduced to stabilize numerical computation. With this 
choice, $\beta^{k}$ obeys a hyperbolic-type equation (for a sufficiently small value of $\Delta t$ ), because the right-hand side of the evolution equation for $F_{l}$ contains vector Laplacian terms as $\beta^{k}{ }_{i i}+\beta^{i}{ }_{i k} / 3$ (e.g. Shibata \& Nakamura 1995; Shibata \& Uryū 2002). The outstanding merit of this gauge condition is that we can save computational time significantly, since we do not have to solve elliptic-type equations. In the numerical computations, we adopted these two spatial gauge conditions, and found that both give the (almost) identical numerical results: As in the case of the AMD gauge condition, the dynamical gauge enables to carry out a longterm stable simulation irrespective of the equations of state. Thus, here, we present numerical results with the dynamical gauge condition to demonstrate its robustness.

During numerical simulations, violations of the Hamiltonian constraint and conservation of mass and angular momentum are monitored as code checks. Several test calculations, including stability and collapse of spherical and rotating neutron stars, as well as convergence tests, have been described in a previous paper (Shibata 2003). Formation of a black hole is determined by finding an apparent horizon.

To model supramassive neutron stars, we adopted the polytropic equations of state of the form

$$
P=K \rho^{1+\frac{1}{n}} .
$$

In this paper, we choose $n=2 / 3,4 / 5,1,3 / 2$ and 2 to systematically study the effects of stiffness of equations of state. During the simulations, we use a $\Gamma$-law equation of state as

$$
P=(\Gamma-1) \rho \varepsilon,
$$

where $\Gamma$ is the adiabatic constant which is set as $1+1 / n$. In the absence of shocks, no heat is generated and the collapse is adiabatic, preserving the polytropic form of the equations of state. This implies that the quantity $P / \rho^{\Gamma}$ measures the efficiency of the shock heating.

As initial conditions, we gave marginally stable and uniformly rotating supramassive neutron stars at massshedding limits in equilibrium states. To induce gravitational collapse, we initially reduced the pressure (i.e., $K$ ) uniformly by $0.5 \%$ in all the simulations. Whenever we reduce the pressure, we solve the equations for the Hamiltonian and momentum constraints to enforce them at $t=0$.

Marginally stable supramassive neutron stars of polytropic equations of state with $2 / 3 \leq n \leq 2$ have the compactness $0.06 \leq M / R \leq 0.25$ (see Table 1 ). Typical compactness of neutron stars is considered to be $\sim 0.15-0.2$ (Shapiro \& Teukolsky 1983; Glendenning 1996). Thus, the present choice of $n$ yields plausible models for marginally stable supramassive neutron stars.

Physical units enter the problem only through the polytropic constant $K$, which can be chosen arbitrarily or else completely scaled out of the problem. Thus, we only display the dimensionless quantities which are defined as

$$
\begin{gathered}
\bar{M}_{*}=M_{*} K^{-n / 2}, \bar{M}=M K^{-n / 2}, \bar{R}=R K^{-n / 2}, \\
\bar{J}=J K^{-n}, \quad \bar{\rho}=\rho K^{n}, \quad \bar{\Omega}=\Omega K^{n},
\end{gathered}
$$

where $M, R$, and $\Omega$ denote the ADM mass, equatorial circumferential radius and the angular velocity. Hereafter, we adopt the units of $K=1$ so that we will omit the bar.
In Table 1, we list the rotating stars at mass-shedding limits that we picked up as initial conditions in the present simulations. All the quantities are scaled to be nondimensional using the relation described in Eq. (8). Stability of uniformly rotating polytropes with $n=1,3 / 2$ and 2 against gravitational collapse has been already studied by Cook et al.(1994). Thus, for these polytropic indices, we choose the stars close to the marginally stable point based on their results. For $n=2 / 3$ and $4 / 5$, we do not know the critical point for the stability. As shown by Cook et al. (1994), however, for stiff equations of state with $n \lesssim 1$, the stability of the uniformly rotating stars at mass-shedding limits changes near a point where the mass is maximum. Thus, we choose the stars of nearly maximum mass along the sequence of the uniformly rotating star at mass-shedding limits.

The ratio of the kinetic energy to the gravitational binding energy for all the stars that we picked up here is much smaller than 0.27 which is a widely believed critical value for onset of the dynamical bar-mode instability in a uniformly rotating star (Chandrasekhar 1969). Thus, the nonaxisymmetric deformation is unlikely to turn on during collapse. This justifies that we assume the axial symmetry.

\section{NUMERICAL RESULTS}

\subsection{Prediction}

Before presenting numerical results, we here predict the plausible outcome of the gravitational collapse. Such prediction helps to understand the reason that a result obtained in a numerical simulation should be the output.

Because of the axial symmetry of the system (and since the fluid is assumed to be inviscid), the mass distribution as a function of the specific angular momentum as well as the baryon rest-mass and angular momentum are conserved throughout the evolution of the system. Using this fact, we can predict the final state of gravitational collapse from the initial condition.

We define the mass distribution as a function of the specific angular momentum according to (e.g., Stark \& Piran 1987)

$$
M_{*}\left(j_{0}\right)=\int_{j \leq j_{0}} d^{3} x \rho_{*},
$$

where $j$ is a value of the specific angular momentum computed as $x \hat{u}_{y}\left(=h u_{\varphi}\right)$ and $j_{0}$ denotes a particular value for $j$. In Fig. 1, we show the mass distribution as a function of the specific angular momentum $M_{*}(j)$ as a function of $j / M$.

To predict the final state of the collapse, we assume that (i) a black hole is formed after the collapse, (ii) most of the mass elements fall into the black hole, and (iii) the value of the nondimensional angular momentum parameter $q \equiv J / M^{2}$ of a formed black hole is nearly equal to the value of the system.

Since the value of $q$ of all the stars that we picked up here is smaller than unity and no heat source exist in the collapsing star, assumption (i) is quite reasonable. (ii) and (iii) are also reasonable because the progenitor of the collapse is uniformly rotating, so that the effect of centrifugal force which prevents a fluid element falling into a black hole is important only around the low-density outer region of the collapsing stars. 
According to (i)-(iii), we assume that the mass and Kerr parameter of the formed black hole are $\approx M$ and $\approx M q$. Around the Kerr black hole, there is the innermost stable circular orbit (ISCO). All the mass elements of circular orbits inside the ISCO have to fall into the black hole. This implies that a mass element of the specific angular momentum which is smaller than the value at the ISCO, $j_{\mathrm{ISCO}}$, has to fall into the black hole.

Assuming that the mass and Kerr parameter of the formed Kerr black holes are $M$ and $M q$, we computed $j_{\text {ISCO }}$ for the models listed in Table 1 using the formula derived by Bardeen et al. (1972). The numerical results are described at the last column of Table 1. In all the models, $j_{\mathrm{ISCO}}$ is larger than $2.5 \mathrm{M}$. (Note that it is $2 \sqrt{3} M$ for $q=0$.) From Fig. 1, we find that the fraction of the mass with $j_{\mathrm{ISCO}}>2.5 \mathrm{M}$ is approximately zero (less than $10^{-3}$ ) irrespective of $n$. Therefore, the final state is predicted to be a Kerr black hole and the disk mass is very small $\left(<10^{-3}\right.$ of the initial stellar mass) for any value of $n$ between $2 / 3$ and 2 .

\subsection{Formation and evolution of black holes}

We performed simulations varying $N$ for a wide range as 180-480. This grid number is several times larger than that in a previous study (Shibata et al. 2000), and enables to check the convergence of the numerical solutions in detail. For $n \leq 3 / 2$, the equatorial radius of marginally stable rotating stars are initially covered by $N / 2$ grid points. The polar radius is covered by $\approx 0.3 N$ in this case. For $n=2$, equilibrium stars have a more centrally-concentrated density configuration than that for stiffer equations of state. To resolve the central region, we arrange the grid spacing with which the equatorial radius is covered by $5 N / 6$ grid points initially. In this case, the polar radius is covered by $\approx N / 2$ grid points.

Numerical computation was in part performed on FACOM VPP5000 in the data processing center of National Astronomical Observatory of Japan, but most of the simulations were carried out using personal computers of Pentium-4 processors, each of which has 2 Gbytes memory and $2.8 \mathrm{GHz}$ clock. Even for $N=480$, it takes only about 5 days to finish a job of $\sim 40000$ time steps on one of these computers.

As a result of the simulations, we found that irrespective of the value of $n$, the collapse proceeds monotonically to be a Kerr black hole. During the collapse, shock heating is negligible in the central region. Namely, $P / \rho^{\Gamma}$ remains approximately constant.

In all the simulations, the apparent horizons were determined in the late phase of the collapse. As reported in a previous paper (Shibata 2003), accuracy of numerical results, measured by the violation of the Hamiltonian constraint, deteriorates monotonically with time, and the computations eventually crashed due to the grid stretching around the black holes. However, the grid number inside the surface of the apparent horizons in this work is large enough to resolve the formation, and evolution of the black hole for a duration $\sim 20 M$ even without black hole excision techniques (W. Unruh 1984, unpublished; Seidel $\&$ Suen 1992). The duration is in general longer with better grid resolutions, and with the largest grid number, we could determine the final state of the collapse approximately. However, to carry out a simulation for more than
$20 M$ after the formation of black holes, excision techniques are absolutely necessary.

We have also checked that the mass distribution as a function of the specific angular momentum is conserved accurately. In Fig. 1(b), we compare the mass distribution at $t=0$ and at the formation of apparent horizon for $n=2$ as an example. The figure shows a good conservation of it.

In Fig. 2, we display the square of the mass of the apparent horizons $M_{\mathrm{AH}}$ as a function of time. Here, $M_{\mathrm{AH}}$ is defined as

$$
M_{\mathrm{AH}} \equiv \sqrt{\frac{A}{16 \pi}}
$$

where $A$ denotes the area of the apparent horizon (e.g., Cook \& York 1990). Figure 2 shows that $M_{\mathrm{AH}}$ approaches an asymptotic value. It is also found that the numerical results are convergent with increase of $N$.

Together with the evolution of $M_{\mathrm{AH}}^{2}$, in Fig. 2, we plot the square of the irreducible mass of the event horizon $M_{\text {irr }}^{2}$ for the Kerr black hole of $M$ and $J$ (dotted horizontal lines) as

$$
M_{\mathrm{irr}}^{2}=\frac{1}{2}\left(M^{2}+\sqrt{M^{4}-J^{2}}\right) .
$$

Here, as $M$ and $J$, we adopt the total values of the system which are computed from the initial data sets. If the final state of the gravitational collapse is a Kerr black hole with negligible disk mass, the mass of the apparent horizon should approach $M_{\text {irr }}$. Figure 2 clearly shows that $M_{\mathrm{AH}}$ asymptotically approaches $M_{\mathrm{irr}}$. Small deviation of the asymptotic value of $M_{\mathrm{AH}}$ from $M_{\mathrm{irr}}$ is likely to be a numerical error. Indeed, with improvement of the grid resolution, the final value of $M_{\mathrm{AH}}$ appears to converge to $M_{\mathrm{irr}}$. With the best resolution, the magnitude of the numerical error in $M_{\mathrm{AH}}$ is less than 1\%. This implies that the final state of the collapse is the Kerr black hole and the fraction of the disk mass is very small (within the magnitude of numerical error $\lesssim 1 \%$ ).

To reconfirm this fact, we display the evolution of the fraction of the mass located outside a coordinate radius $r_{0}, M_{*}\left(r>r_{0}\right) / M_{*}$, for $n=2$ (solid curve), $3 / 2$ (dotted curve), 1 (dashed curve), and $2 / 3$ (long-dashed curve) in Fig. 3. The result for $n=4 / 5$ is essentially the same, so that we omit it. Here, $r_{0}$ is chosen as $\approx 0.6 M$, which is approximately equal to the asymptotic value of the coordinate polar-radius of the apparent horizon in the present computations. We found that in our gauge, the coordinate equatorial-radius is slightly larger than the polarradius, so that the fraction of the mass outside the apparent horizon is slightly smaller than $M_{*}\left(r>r_{0}\right) / M_{*}$. Figure 3 shows that before the collapse, most of the fluid elements are located outside $r=r_{0}$, but during the collapse, $M_{*}\left(r>r_{0}\right) / M_{*}$ monotonically decreases and approaches zero. This confirms the results in Fig. 2. The present results also reconfirm the same conclusion reached previously in less-resolved simulations for $n=1$ (Shibata et al. 2000).

We note that for $n=2$, a small amount of mass elements appears to be outside the black hole at the termination of the simulations. We infer that they will be eventually swallowed into the black hole because the specific angular 
momentum for the fluid element is not large enough to form disks around the black hole.

Recall that we study the collapse of uniformly rotating stars of maximum angular velocity, implying that the effect of rotation is taken into account most efficiently. Therefore, we conclude that the final state after the collapse of all the marginally stable and uniformly rotating polytropic stars with $2 / 3 \leq n \leq 2$ is a Kerr black hole and the disk mass is $<10^{-3} M_{*}$.

It should be noted that for smaller value of $n, M_{\mathrm{AH}}$ reaches $M_{\text {irr }}$ more quickly. For $n=2 / 3$, the growth timescale of $M_{\mathrm{AH}}$ from 0 to $\sim M_{\text {irr }}$ is $\sim 6 M$ while for $n=2$, it is larger than $30 M$. This reflects that the nature of the collapse depends strongly on the initial density configuration which is determined by the stiffness of the equations of state. For stiffer equations of state, the density of the initial condition distributes rather uniformly. Thus, the collapse proceeds coherently. For softer equations of state, on the other hand, the initial condition has more centrally-concentrated density distribution with lowdensity outer envelops. Thus, the central region collapses to a black hole earlier, and then the outer region falls into the black hole spending a longer timescale than that for stiffer equations of state.

To illustrate that the identical numerical results were obtained in two different spatial gauge conditions, in Fig. 4 , we display evolution of $\phi$ and $\rho_{*}$ at $r=0$ as well as $M_{\mathrm{AH}}^{2}$ for $n=3 / 2$ with $N=360$. The solid and dotted curves denote the numerical results in the dynamical and AMD gauge conditions, respectively. We see that both results are in good agreement.

Finally, we address the following point: The collapse of compact stars to black holes is among the most interesting processes leading to the production of gravitational waves. As pointed out by Stark and Piran (1985), quasinormal modes of a black hole would be excited after the formation, and as a result, gravitational waves associated with such quasinormal-mode oscillations may be emitted. It is an interesting subject to clarify how large the amplitude of gravitational waves is. From this motivation, we tried to extract gravitational waves in the simulation, but we were not able to do because the amplitude is likely much smaller than the typical size of numerical noise of our present simulation. The reason that the amplitude is very small is the following: (i) the collapse coherently proceeds, i.e., almost all the fluid elements collapse to form a black hole simultaneously. In such case, the excitation of the quasinormal modes of a black hole is likely to be weak, because the quasinormal modes are excited by perturbations struck after formation of a black hole; (ii) the nondimensional angular momentum parameter $q$ is not very large $<0.7$. As Stark and Piran showed that a large amount of gravitational waves is emitted for $q$ close to 1 .

\section{SUMMARY AND DISCUSSION}

We have reported new numerical results of axisymmetric simulations for the gravitational collapse of rapidly and uniformly rotating supramassive neutron stars to black holes in full general relativity. The initial conditions for the neutron stars are given using polytropic equations of state for a wide range of the polytropic index as $n=2 / 3$, $4 / 5,1,3 / 2$ and 2 . The initial state of the rotating stars is marginally stable against the quasiradial gravitational collapse and at the mass-shedding limit. The hydrodynamic simulations were carried out using a high-resolution shock-capturing scheme with the $\Gamma$-law equations of state. We have demonstrated that irrespective of the value of $n(2 / 3 \leq n \leq 2)$, the collapse monotonically proceeds with negligible shock heating, and the final state is a Kerr black hole with a small fraction of the disk mass.

As mentioned in Sec. 3.1, the results obtained in this paper can be expected from the initial conditions. In the same manner, we can expect the final states of the gravitational collapse for softer equations of state with $n>2$. With a large value of $n \sim 3$, we may model an unstable massive stellar core at the final stage of stellar evolution and a supermassive star of $M \gtrsim 10^{5} M_{\odot}$. In Fig. 5, we show the mass distribution as a function of the specific angular momentum of the marginally stable and uniformly rotating stars at mass-shedding limits for $n=2.5,2.9$, and 3 . The marginally stable stars for these polytropic indices have been already determined by Cook et al. (1994) for $n=2.5$ and 2.9 and Baumgarte and Shapiro (1999) for $n=3$. The nondimensional angular momentum parameter $q$ is $\approx 0.39,0.57$, and 0.96 for $n=2.5,2.9$ and 3 , so that $j_{\mathrm{ISCO}} / M$ for a black hole of mass $M$ and angular momentum $J$ is $\approx 3.0,2.8$, and 1.8 , respectively. From Fig. 5, we can expect that the final state after the collapse for $n=2.5$ is a Kerr black hole and only a small fraction of the initial stellar elements $\left(\sim 10^{-3} M_{*}\right)$ forms the disks. On the other hand, disks of mass of $\gtrsim 0.01 M_{*}$ and $\gtrsim 0.1 M_{*}$ are likely to be formed for $n=2.9$ and 3 , respectively. (If the mass of the black holes is smaller than $M, j_{\text {ISCO }}$ is also smaller and, hence, the disk mass could be larger. This implies that the numerical fraction mentioned here is the minimum value.) The same conclusion for $n=3$ has been already drawn by Shibata and Shapiro (2002) and Shapiro and Shibata (2002) in a more careful analysis.

The reason why disks are formed for $n \gtrsim 2.9$ is simply that the marginally stable stars with polytropic equations of state of such large value of $n$ have a large equatorial radius with $R / M \gtrsim 200$, and hence the specific angular momentum for a certain fraction of the fluid elements is large enough to escape from swallowing into a black hole. The present study together with the previous one (Shibata \& Shapiro 2002) shows that nature of the collapse of rapidly rotating stars to a black hole depends strongly on the equations of state in particular for $n \sim 3$.

This work was supported by Japanese Monbukagakusho Grants (Nos. 13740143, 14047207, 15037204 and 15740142).

\section{REFERENCES}

Alcubierre, M. et al., 2001, Phys. Rev, D 64, 061501.

Alcubierre, M. et al., 2001, Int. J. Mod. Phys. D 10, 273.

Bardeen, J. M., Press, W. H. and Teukolsky, S. A., 1972, ApJ, 178,

347.
Baumgarte, T. W. \& Shapiro, S. L., 1999, ApJ, 526, 941.

Baumgarte, T. W., Shapiro, S. L. \& Shibata, M., 2000, ApJ, 528, L29. 
TABLE 1

PARAMETERS OF THE initial CONDitions

\begin{tabular}{cccccccccc}
\hline \hline$n$ & $\rho_{c}$ & $M_{*}$ & $M$ & $M / R$ & $\Omega$ & $J / M^{2}$ & $T /|W|$ & $\alpha(r=0)$ & $j_{\mathrm{ISCO}} / M$ \\
\hline $2 / 3$ & 0.730 & 0.184 & 0.158 & 0.248 & 0.791 & 0.670 & 0.117 & 0.329 & 2.64 \\
\hline $4 / 5$ & 0.520 & 0.190 & 0.168 & 0.212 & 0.572 & 0.626 & 0.102 & 0.382 & 2.71 \\
\hline 1 & 0.296 & 0.206 & 0.188 & 0.175 & 0.392 & 0.561 & 0.0809 & 0.442 & 2.82 \\
\hline $3 / 2$ & 0.0570 & 0.304 & 0.290 & 0.106 & 0.117 & 0.450 & 0.0465 & 0.607 & 2.97 \\
\hline 2 & 0.00523 & 0.559 & 0.549 & 0.0643 & 0.0242 & 0.388 & 0.0268 & 0.750 & 3.05 \\
\hline
\end{tabular}

Note. - The central density $\rho_{c}$, baryon rest-mass $M_{*}$, ADM mass $M$, compactness $M / R$, angular velocity, angular momentum $J$ in units of $M^{2}$, ratio of the kinetic energy to the gravitational binding energy, and central value of the lapse function. Here, $R$ denotes the circumference radius at the equatorial surface. The last column shows the specific angular momentum of a test particle orbiting a Kerr black hole of mass $M$ and angular momentum $J$. All the quantities are shown in units of $c=G=K=1$.

Chandrasekhar, S., Ellipsoidal Figures of Equilibrium (Yale University Press, New Haven, Connecticut, 1969).

Cook, G. B. \& York, J. W., 1990, Phys. Rev. D 41, 1077.

Cook, G., Shapiro, S. L. and Teukolsky, S. A., 1992, ApJ. 398, 203. 1994a, ApJ. 422, 227.

- 1994b, ApJ. 423, L117.

Font, J. A., 2000, Liv. Rev. Relativ. 3, 2.

Font, J. A., et al., 2002, Phys. Rev. D 65, 084024.

Friedman, J. L., Ipser, J. R. and Sorkin, R. D., 1988, ApJ. 325, 722.

Glendennig, N. K., 1996, Compact Stars (A \& A Library, Springer)

Komatsu, H., Eriguchi, Y. \& Hachisu, I., 1989 Mon. Not. R. astr. Soc., $237,355$.

Lindblom, L \& Schell, M. A., 2003, gr-qc/0301120.

Nakamura, T., 1981, Prog. Theor. Phys. 65, 1876.

Nakamura, T., Oohara, K., \& Kojima, Y., 1987, Prog. Theor. Phys. Supple. 90, 1 .

Piran, T. \& Stark, R. F., in Dynamical Spacetimes and Numerical Relativity, ed. J. M. Centrella (Cambridge University Press, 1986), 40.

Salgado, M., Bonazzola, S., Gourgoulhon, E. \& Haensel, P., 1994, A \& Ap. 291, 155.

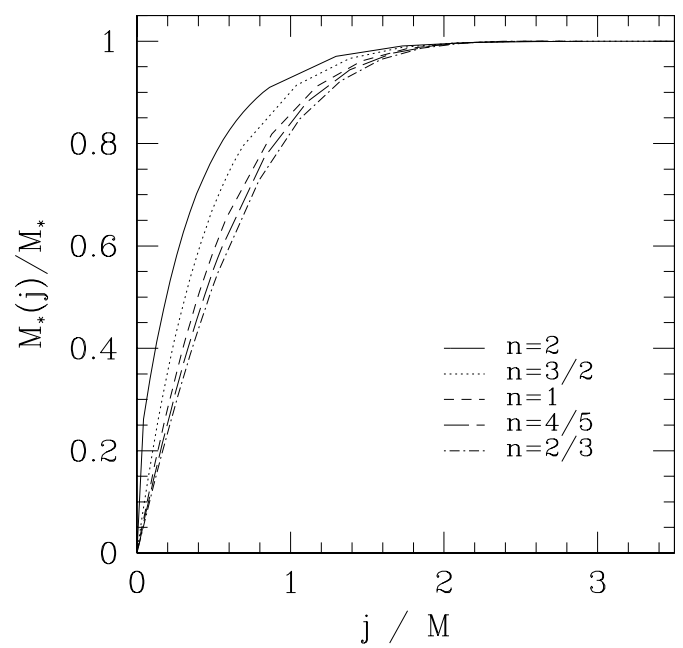

(a)

(b)

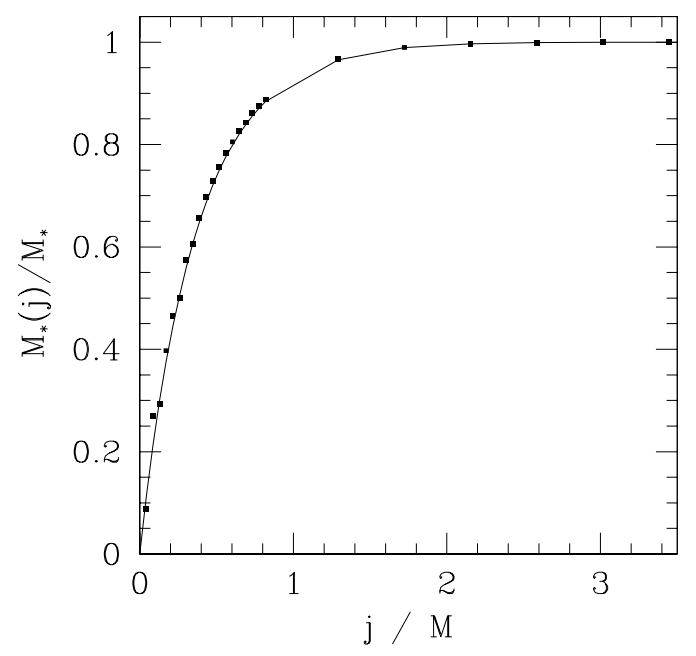

Fig. 1.- (a) The mass distribution as a function of the specific angular momentum for $n=2 / 3-2$ at $t=0$. (b) The same as (a) but at $t=0$ (solid curves) and at the formation of the apparent horizon (filled circles) for $n=2$. This is the result for a simulation with $N=480$.
Seidel, E. \& Suen, W., 1992, Phys. Rev. Lett. 69, 1845.

Siebel, F., Font, J. A., Müller, E., Papadopoulos, P., 2002, Phys. Rev. D 65, 064038. 2003, Phys. Rev. D submitted.

Shapiro, S. L. \& Teukolsky, S. A., 1983, Black Holes, White Dwarfs, and Neutron Stars (Wiley interscience, New York).

Shapiro, S. L. \& Shibata, M., 2002, ApJ. 577, 904.

Shibata, M., 1999a, Prog. Theor. Phys. 101, 1199.

. 1999b, Phys. Rev. D 60, 104052.

- 2000, Prog. Theor. Phys., 104, 325

. 2003, Phys. Rev. D 67, 024033.

Shibata, M., Baumgarte, T. W., \& Shapiro, S. L. 2000, Phys. Rev.

D 61, 044012.

Shibata, M. \& Nakamura, T., 1995, Phys. Rev. D 52, 5428.

Shibata, M. and Shapiro, S. L., 2002, ApJ. 572, L39.

Shibata, M. and Uryū, K., 2000, Phys. Rev. D 61, 64001

. 2002, Prog. Theor. Phys. 107, 265.

Stark, R. F. \& Piran, T., 1985, Phys. Rev. Lett. 55, 891.

1987, Comp. Phys. Rep., 5, 221

Stergioulas, N., 1998, Liv. Rev. Relativ. 1, 8. 


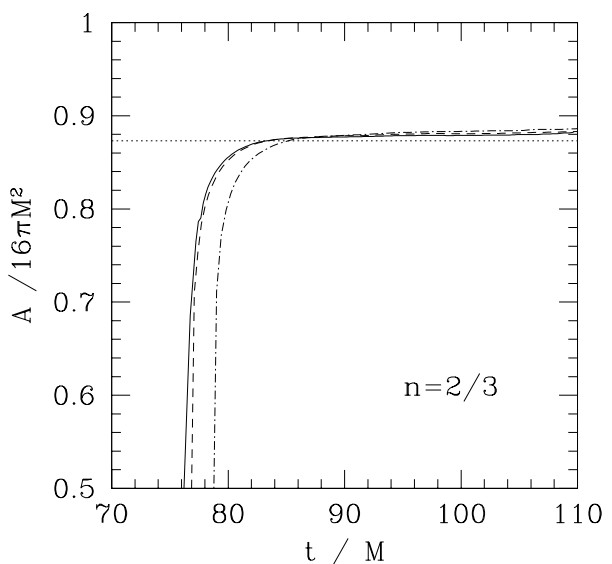

(a)

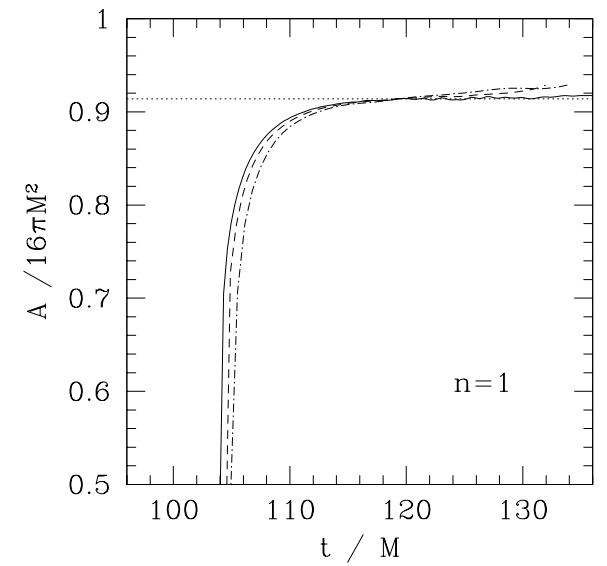

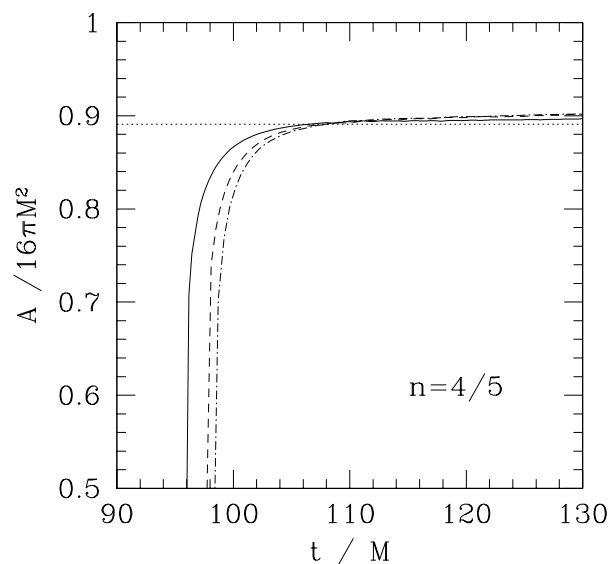

(b)

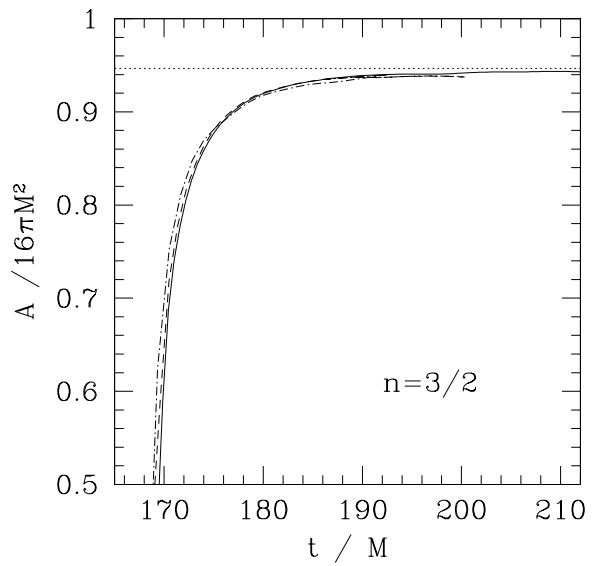

(c)

(d)

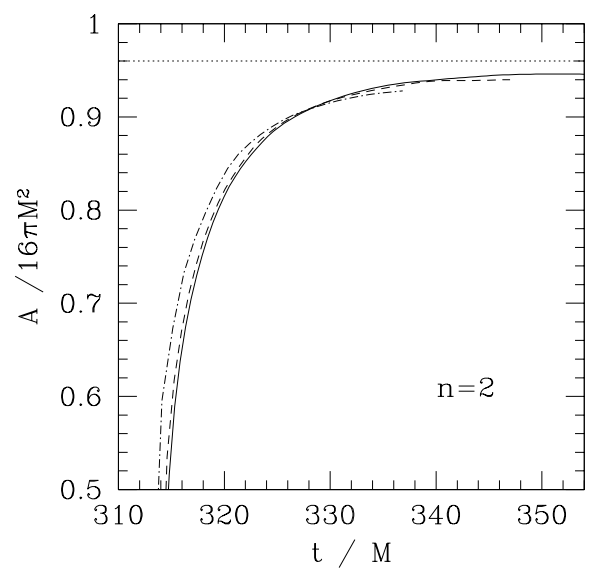

FIG. 2. - Evolution of the area of the apparent horizon in units of $16 \pi M^{2}$ (i.e., the square of the apparent horizon mass) for (a) $n=2 / 3$, (b) $4 / 5$, (c) 1 , (d) $3 / 2$ and (d) 2 . For (a)-(d), the solid, dashed and dotted-dashed curves are results for $N=360,240$, and 180, and for (e), the solid and dashed curves are results for $N=480,360$, and 240. The dotted horizontal lines denote the area of the event horizon for Kerr black holes of a given set of $J$ and $M$ for which we adopt the values of the initial conditions. 


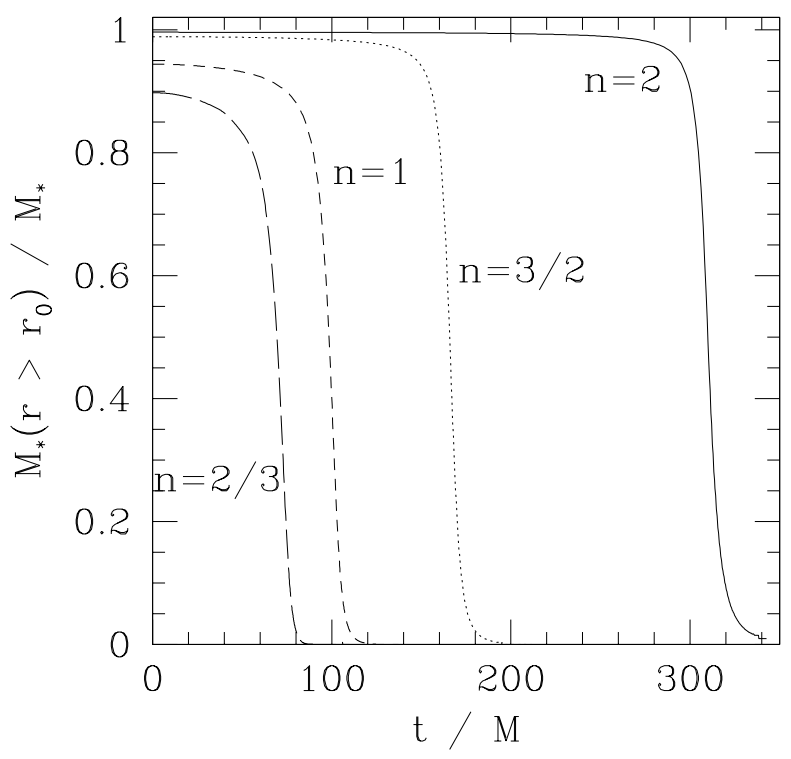

FIG. 3. - Evolution of the total mass outside a coordinate radius $r_{0}$ for $n=2$ (solid curve), $3 / 2$ (dotted curve), 1 (dashed curve), and $2 / 3$ (long-dashed curve). $r_{0}$ is chosen as $\sim 0.6 M$ which is approximately equal to the asymptotic value of the coordinate polar-radius of the apparent horizon in the present computations. To plot the curves, we choose the numerical results with $N=360$. The time is shown in unit of $M$.

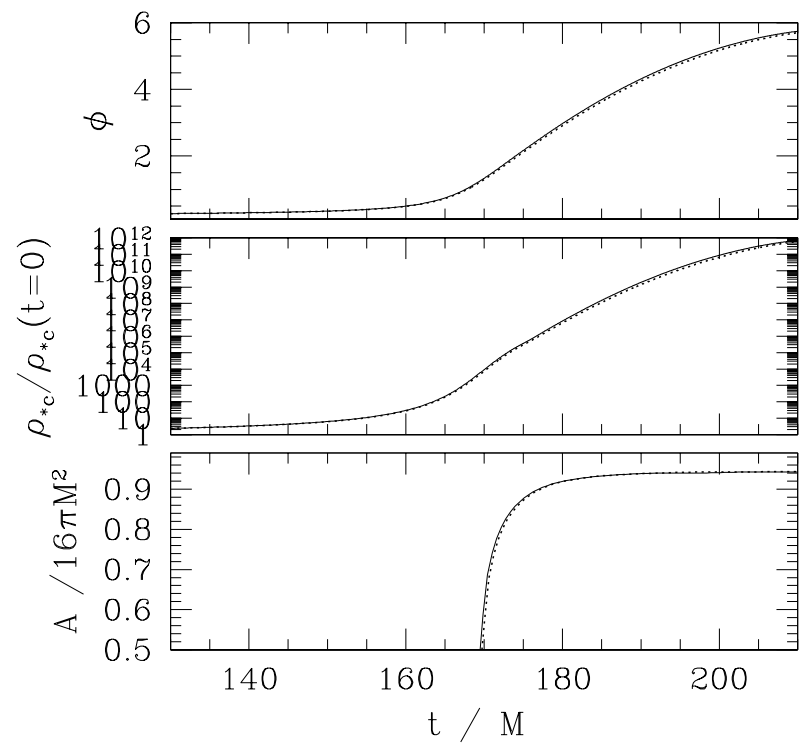

FIG. 4. - Evolution of $\phi$ and $\rho_{*}$ at $r=0$ as well as $M_{\mathrm{AH}}^{2}$ for $n=3 / 2$ with $N=360$. The solid and dotted curves denote the results by the dynamical and AMD gauge conditions, respectively, and they approximately coincide. 


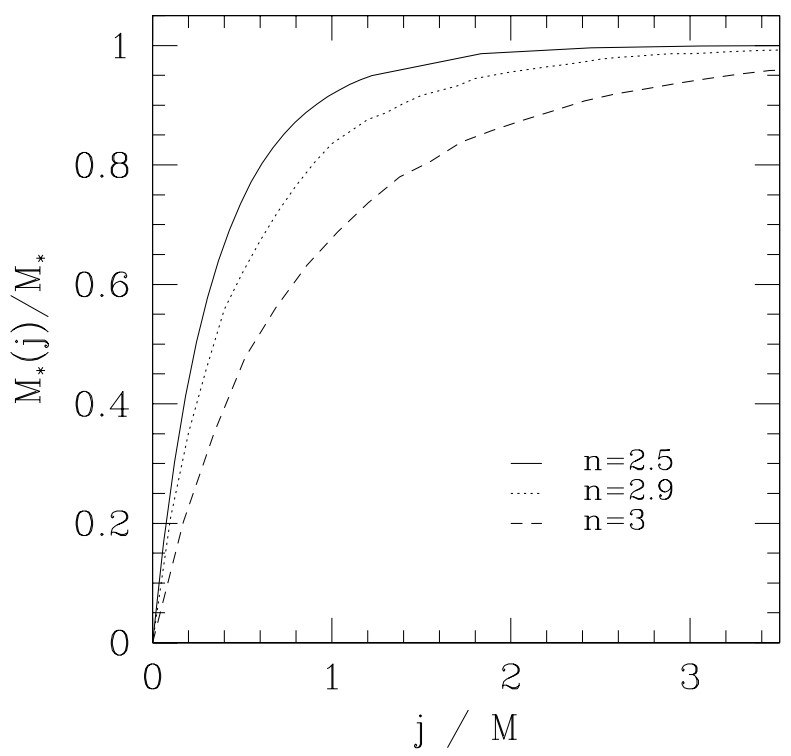

FIG. 5.- The mass distribution as a function of the specific angular momentum for $n=2.5,2.9$, and 3 . 